\title{
UTILIZAÇÃO DE GABAPENTINA PARA MANEJO CLÍNICO DE DOR NEUROPÁTICA EM CÃES - RELATO DE CASO
}

\author{
Suelen Chaiane Sontag ${ }^{1}$ \\ Salviano Tramontin Belletini ${ }^{2}$ \\ Rafael Santos Tramontin ${ }^{2}$ \\ Juliano Bortolo De Conti ${ }^{3}$ \\ Evandra Maria Voltarelli Pachaly ${ }^{4}$ \\ Ana Maria Quessada ${ }^{5}$ \\ Natália Saraiva Rodrigues ${ }^{6}$ \\ José Ricardo Pachaly ${ }^{5}$
}

SONTAG, S. C.; BElletini, S. T.; TRAMONTIN, R. S.; CONTI, J. B. de; PACHALY, E. M. V.; QUESSADA, A. M.; RODRIGUES, N. S.; PACHALY, J. R. Utilização de gabapentina para manejo clínico de dor neuropática em cães - Relato de caso. Arq. Ciênc. Vet. Zool. UNIPAR, Umuarama, v. 20, n. 4, p. 221-231, out./dez. 2017.

\begin{abstract}
RESUMO: Este artigo revisa a literatura sobre o uso da gabapentina e relata seu uso para tratamento e manejo clínico de um caso, dor neuropática em uma cadela da raça Schnauzer miniatura com idade de nove anos e massa corporal de 8,0 kg. Havia histórico de dor e claudicação, inicialmente nos membros torácicos e a seguir nos membros pélvicos. Após exame físico, exame neurológico e exames de laboratório e imagem, não se observou qualquer sinal de alteração óssea ou articular, sendo descartada a possibilidade de artrite e/ou artrose. Como o problema continuava a evoluir, a suspeita foi centrada na possibilidade de dor neuropática, definida como dor crônica causada por uma consequência direta de lesão ou disfunção dos axônios ou corpos dos neurônios, capaz de causar interrupção da bainha de mielina tanto em sistema nervoso periférico quanto central. Diagnosticar a dor em animais, que não possuem intelecto e são incapazes de falar, não é uma tarefa fácil, e na dor neuropática o problema é ainda maior. É difícil identificar quando os animais sentem sensações como formigamento, queimação e agulhamento, comuns na dor neuropática. Boa anamnese pode fornecer informações importantes para diagnosticar se um paciente está ou não vivenciando dor neuropática, e assim indicar tratamento específico. A dor neuropática costuma responder de forma insuficiente aos analgésicos comuns, sendo alguns fármacos anticonvulsivantes os principais agentes terapêuticos viáveis para esse tipo de dor, seja de origem central ou periférica. A gabapentina, anticonvulsivante de segunda geração, é uma substância análoga ao ácido $\gamma$-aminobutírico (GABA) com uma boa penetração na barreira hematoencefálica. É um dos novos fármacos antiepiléticos utilizados em pessoas, e só recentemente passou a ser empregada em cães, sendo atualmente utilizada com sucesso como adjuvante no tratamento de processos dolorosos crônicos e neuropáticos. No caso apresentado, o uso clínico da gabapentina foi eficaz no controle da dor neuropática, com importante redução dos sinais de dor e notória melhoria na qualidade de vida da paciente. Trata-se de uma opção terapêutica de custo baixo e fácil utilização, com baixo índice de efeitos adversos.
\end{abstract}

PALAVRAS-CHAVE: Analgesia. Analgésicos. Anticonvulsivantes. Cães. Dor.

\section{GABAPENTINE FOR CLINICAL MANAGEMENT OF NEUROPATHIC PAIN IN DOGS - CASE REPORT}

\begin{abstract}
This paper reviews the literature on gabapentin and reports its use for treatment and clinical management in a case of neuropathic pain diagnosed in a nine-year-old miniature Schnauzer weighing $8.0 \mathrm{~kg}$. The chief complaint concerned the history of pain and lameness, initially in the forelimbs and thereafter in the hind limbs. After physical examination, neurological examination, laboratory tests and image screening, no signals of bone or joint changes were observed, thus discarding the possibility of arthritis and/or osteoarthritis. Since the morbid condition continued to evolve, the clinical suspicion was focused on the possibility of neuropathic pain, defined as chronic pain caused by a direct consequence of injury or dysfunction of axons or bodies of neurons, causing disruption of the myelin sheath on both the peripheral and central nervous system. The diagnostic of pain in animals that lack intellect and are unable to speak is not an easy task, and with neuropathic pain the problem is even greater. It is difficult to identify when animals feel sensations such as tingling, burning and needling, common in neuropathic pain. Good history can provide important information to diagnose whether a patient is or is not experiencing neuropathic pain, and thus indicate specific treatments. Neuropathic pain tends to respond inadequately

DOI: 10.25110 /arqvet.v20i4.2017.6401

${ }^{1}$ Mestranda do Programa de Pós Graduação em Produção Sustentável e Saúde Animal da Universidade Estadual de Maringá - UEM, Câmpus Umuarama PR. E-Mail: suh_sontag@hotmail.com

${ }^{2}$ Doutorandos do Programa de Pós Graduação em Ciência Animal da Universidade Paranaense - UNIPAR, Câmpus Umuarama - PR. E-Mail: salviano@ prof.unipar.br; rafael_tramontin@hotmail.com

${ }^{3}$ Professor do Programa de Pós Graduação em Produção Sustentável e Saúde Animal da Universidade Estadual de Maringá - UEM, Câmpus Umuarama PR. E-Mail: julianodeconti@yahoo.com.br

${ }^{4}$ Professora do Curso de Graduação em Medicina Veterinária da Faculdade Integrado de Campo Mourão - PR. E-Mail: emvoltarelli@hotmail.com ${ }^{5}$ Professores do Programa de Pós Graduação em Ciência Animal da Universidade Paranaense - UNIPAR, Câmpus Umuarama - PR. E-Mail: mariaquessada@ prof.unipar.br; pachaly@uol.com.br

${ }^{6}$ Bolsista PIBIC, Graduanda do Curso de Medicina Veterinária da Universidade Paranaense - UNIPAR, Câmpus Umuarama - PR. E-Mail: nataliasrodrigues123@gmail.com
\end{abstract}


to common analgesics, with some anticonvulsant drugs being the main viable therapeutic agents for that kind of pain, whether of central or peripheral origin. Gabapentin, a second-generation antiepileptic substance, is analogous to $\gamma$-aminobutyric acid (GABA), with good penetration in the blood-brain barrier. It is one of the new antiepileptic drugs used in humans, and it has only recently started to be employed in dogs, being currently successfully used as an adjunct in the treatment of chronic and neuropathic pain processes. In this particular case, the clinical use of gabapentin was effective in controlling neuropathic pain, with significant reduction of pain signals and marked improvement in the quality of life of the patient. It is a relatively low-cost treatment option and easy to be used by pet owners, with a low rate of adverse effects.

KEYWORDS: Analgesia. Analgesics. Anticonvulsants. Dogs. Pain.

\section{UTILIZACIÓN DE GABAPENTINA PARA MANEJO CLÍNICO DE DOLOR NEUROPÁTICO EN PERROS - RELATO DE CASO}

RESUMEN: Este artículo revisa la literatura sobre el uso de gabapentina y relata su uso para tratamiento y manejo clínico de un caso de dolor neuropático en una perra de la raza Schnauzer miniatura con edad de nueve años y masa corporal de $8,0 \mathrm{~kg}$. Había histórico de dolor y claudicación, inicialmente los miembros torácicos y a seguir los miembros pélvicos. Tras examen físico, examen neurológico y exámenes de laboratorio e imagen, no se observó cualquier señal de alteración ósea o articular, siendo descartada la posibilidad de artritis o artrosis. Como el problema continuaba a evolucionar, la sospecha se centró en la posibilidad de dolor neuropático, definida como dolor crónica causada por una consecuencia directa de lesión o disfunción de los axones o cuerpos de las neuronas, capaz de causar interrupción de la vaina de mielina, tanto en el sistema nervioso periférico como central. Diagnosticar el dolor en animales, que no poseen intelecto y son incapaces de hablar no es una tarea fácil, y en el dolor neuropático aún es peor. Es difícil identificar cuando los animales sienten sensaciones como hormigueo, quemazón y agujetas, comunes en el dolor neuropático. Una buena anamnesis puede proporcionar importantes informaciones para diagnosticar si un paciente está o no vivenciando dolor neuropático, y así indicar tratamiento específico. El dolor neuropático puede responder de forma insuficiente a los analgésicos comunes, siendo algunos fármacos anticonvulsivantes los principales agentes terapéuticos viables para ese tipo de dolor, sea de origen central o periférico. La gabapentina, anticonvulsivante de segunda generación, es una sustancia análoga al ácido $\gamma$-aminobutírico (GABA) con buena penetración en la barrera hematoencefálica. Es uno de los nuevos fármacos antiepilépticos utilizados en personas, y solo recientemente pasó a ser empleada en perros, siendo actualmente utilizada con suceso como adyuvante en el tratamiento de procesos dolorosos crónicos y neuropáticos. En el caso presentado, el uso clínico de la gabapentina fue eficaz en el control del dolor neuropático, con importante reducción de los señales de dolor y notoria mejora en la calidad de vida del paciente. Se trata de una opción terapéutica de bajo costo y de fácil utilización, con bajo índice de efectos adversos.

PALABRAS CLAVE: Analgesia. Analgésicos. Anticonvulsivantes. Perros. Dolor.

\section{Introdução}

Durante muito tempo o homem alimentou a crença de que os animais são incapazes de sentir dor, não necessitando de analgesia ao serem submetidos à vivissecção. Esse pensamento perdurou até o século passado e, atualmente, apesar dos avanços ocorridos, os animais ainda não recebem analgesia adequada. Muitos médicos veterinários ainda acreditam que a dor faça parte do processo de cura, entendendo como natural que o paciente submetido a um procedimento cirúrgico ou portador de uma doença crônica sinta dor. Muitos fatores contribuem para isso, incluindo o desconhecimento dos efeitos deletérios da dor sobre os diferentes sistemas, o receio dos efeitos adversos da administração de determinados fármacos potentes, a desinformação por parte de alguns profissionais e até mesmo a falta de preocupação com o bem-estar animal (FANTONI; MASTROCINQUE, 2010).

Segundo a Associação Internacional para o Estudo da Dor, dor é uma experiência sensorial e emocional desagradável, associada a lesão tissular real ou potencial, ou descrita em termos de tal lesão (MERSKEY; BOGDUK, 1994). Essa percepção baseia-se não apenas no aporte neuronal, como também inclui a interpretação desse aporte com base na experiência passada e no estado mental atual (JOHNSTON, 2004). Já para Kitchell (1987), numa definição mais ampla, a dor em animais é uma experiência emocional e sensorial aversiva, uma percepção que induz ações motoras protetoras e pode modificar as características da espécie, inclusive o comportamento social.

A dor pode ser localizada ou generalizada, superficial ou profunda, local ou referida, e ter origem visceral, somática, neuropática ou psicogênica, e de acordo com a duração, pode ser aguda ou crônica. Pode modificar-se de acordo com fatores como postura, atividade física, estado de atenção, emoções, temperatura e umidade ambiental, por exemplo (CAPONERO; VIEIRA; TEIXEIRA, 2001). Dor induz respostas reflexas que resultam no aumento do tônus simpático, vasoconstrição, aumento da resistência vascular sistêmica, aumento do débito cardíaco pelo aumento da frequência cardíaca, aumento do consumo de oxigênio pelo miocárdio, diminuição do tônus gastrintestinal e urinário e aumento do tônus musculoesquelético (MATHEWS, 2005).

A dor neuropática ou neurogênica é um processo crônico produzido por dano ao tecido nervoso, consequência direta de lesão ou disfunção dos axônios ou corpo dos neurônios, que causa interrupção da bainha de mielina tanto no sistema nervoso periférico quanto central (BACKONJA, 2003). Caracteriza-se pela aparição de hiperalgesia, dor espontânea, parestesia e alodinia mecânica e por frio (PISERA, 2005; SCHAIBLE, 2006). No caso da dor neuropática ou mista, a eficácia dos analgésicos de uso comum pode ser limitada, e recomendam-se fármacos de diversas classes terapêuticas, incluindo opioides (como morfina e tramadol), antagonistas NMDA (como a cetamina), antidepressivos tricíclicos (como amitriptilina e nortriptilina) e anticonvulsivantes (como gabapentina e pregabalina) (AGS, 2002; MALANGA; PAS- 
TER, 2007).

A gabapentina é um dos novos fármacos antiepiléticos utilizados em pessoas, e mais recentemente em cães, sendo empregada com sucesso como adjuvante no tratamento de processos dolorosos crônicos e neuropáticos (STOELTING, 1999; MARCOLIM; TATSCH, 2000).

\section{Revisão de Literatura}

\section{Fisiologia da dor}

A dor foi conceituada pela primeira vez em 1986, pela Associação Internacional para o Estudo da Dor, como uma experiência sensorial e emocional desagradável que está associada à lesões reais ou potenciais (MERSKEY; BOGDUK, 1994). O termo nocicepção está relacionado com o reconhecimento de sinais dolorosos pelo sistema nervoso, que formula informações relacionadas à lesão. Baseado nesses conceitos, o termo dor seria melhor aplicado à pessoas do que aos animais, por envolver um componente emocional. Mesmo assim, tornou-se uma convenção o uso do termo "dor" para pacientes humanos e animais (HELLEBREKERS, 2002). A Agência Americana de Pesquisa e Qualidade em Saúde e a Sociedade Americana de Dor descrevem a dor como o quinto sinal vital (SOUSA, 2002), devendo sua presença ser registrada juntamente com pressão arterial, temperatura, frequência cardíaca e frequência respiratória, os quatro sinais vitais primários (SILVA, 2010).

Para ocorrer sucesso no tratamento de processos dolorosos, é necessária avaliação cuidadosa de sua natureza, entendimento dos diferentes tipos e padrões de dor e conhecimento do arsenal terapêutico, sendo que a boa avaliação inicial da dor é a base para as intervenções terapêuticas.

Para aliviar efetivamente a dor em animais, é necessário conhecimento fisiológico detalhado sobre as diferentes vias da dor, os mediadores químicos e os tipos de receptores nela envolvidos. Só assim é possível estabelecer protocolos de tratamento adequados ao seu alívio máximo (HELLEBREKERS, 2002).

Os nervos periféricos podem ser considerados uma extensão do sistema nervoso central (SNC), consistindo de fibras nervosas sensoriais, motoras e autônomas. Atuam como condutores elétricos pelos quais as informações sensoriais e motoras são transmitidas. Os terminais das fibras nervosas sensoriais reconhecem e transformam vários estímulos ambientais em sinais elétricos (potenciais de ação) que são transmitidos ao corno dorsal da medula espinhal, onde são alterados e retransmitidos ao tronco encefálico e ao cérebro, que interpreta e produz a sensação de dor (MUIR III; GAYNOR, 2009). Esses terminais nervosos são terminações livres não mielinizadas, denominadas nociceptores (PATEL, 2010).

A via nociceptiva inclui nociceptores periféricos, fibras nervosas livres em nervos periféricos, medula espinhal, do tronco encefálico, vias espinhais e cerebrais, e áreas de processamento central no tronco encefálico, no tálamo e no córtex cerebral (LORENZ; KORNEGAY, 2006). A dor é iniciada pela estimulação dos nociceptores, encontrados em quase todos os tecidos, porém mais numerosos naqueles que interagem com o ambiente, como pele, músculos e articulações, em comparação com os tecidos mais protegidos, como as vísceras (JOHNSTON, 2004). Os nociceptores são inervados por dois tipos de nervos aferentes, as fibras mielinizadas $\mathrm{A} \delta$ e as não mielinizadas $\mathrm{C}$. A estimulação de fibras do tipo $\mathrm{C}$ induz liberação de mediadores inflamatórios e neurotransmissores no corno dorsal da medula espinhal, exacerbando a percepção da dor, fenômeno conhecido como sensibilização central (JOHNSTON, 2004).

A partir da periferia, as fibras aferentes adentram o canal medular, por meio da raiz dorsal, como nervos periféricos. Por essas fibras, o impulso ascende para o tálamo e o córtex cerebral, onde se processam a integração e a interpretação do aporte nociceptivo (JOHNSTON, 2004). A ativação dessas vias leva à sensação de dor

\section{Classificação da dor}

A vantagem de classificar a dor é fornecer informações sobre sua possível origem e possibilitar o planejamento farmacoterapêutico apropriado. Por exemplo, síndromes neuropáticas dolorosas geralmente respondem à medicações adjuvantes, como antidepressivos e anticonvulsivantes, e em dores nociceptivas, a melhor indicação é o uso de anti-inflamatórios não esteroides, isoladamente ou em combinação com opióides (DUARTE, 1998).

Quanto à origem, a dor pode ser classificada como fisiológica ou patológica (THURMON; TRANQUILLI.; BENSON, 1999). A dor fisiológica é um sistema de alerta que auxilia na proteção do organismo contra danos nos tecidos por ameaças físicas, térmicas ou químicas (WOOLF; SALTER, 2000). Inicia por ativação de receptores (nociceptores) de alto limiar por danos teciduais, sendo bem delimitada e transitória, e acompanhada de reflexos protetores que limitam o dano potencial da agressão (LAMONT; TRANQUILLI; GRIMM, 2000). Já a dor patológica caracteriza-se pela transformação de um estímulo não lesivo em lesivo, desencadeando sensibilidade dolorosa (alodinia), resposta exagerada aos estímulos nóxicos (hiperalgesia) e aumento da área de hipersensibilidade (hiperalgesia secundária) (FANTONI; MASTROCINQUE, 2004).

Em termos neurofisiológicos, a dor pode ser diferenciada pelos mecanismos básicos que fazem a mediação em sua origem, sendo classificada como de origem nociceptiva ou não-nociceptiva (ALMEIDA et al., 2006).

A dor nociceptiva ocorre por ativação fisiológica de receptores ou da via dolorosa, e está relacionada à lesões em tecidos ósseos, musculares ou ligamentares (BENNET et al., 2006). Resulta da ativação das terminações nervosas sensitivas delta $\mathrm{A}$ e C (nociceptores) por estímulos mecânicos, térmicos ou químicos. Esses processos podem ser sensibilizados por substâncias algogênicas, resultando em hiperalgesia (TRANQUILLI, 2004). Segundo Souza et al. (2001), a dor nociceptiva pode ser dividida em somática ou visceral. A dor somática origina-se de estruturas superficiais, como pele, tecido subcutâneo e musculatura. Duarte (1998) descreveu a dor nociceptiva somática como uma sensação dolorosa rude, que pode ser exacerbada por movimentos (dor incidente) e aliviada pelo repouso. É localizada e variável, conforme a lesão básica, e alguns exemplos de dores nociceptivas somáticas são dores pós-operatórias, musculoesqueléticas e artríticas. Já a dor visceral origina-se das vísceras abdominais e torácicas, sendo associada a princípio à irritação das superfí- 
cies serosas (SOUZA et al., 2001), sendo definida por Duarte (1998) como a dor difusa, profunda, opressiva e constritiva provocada por distensão de vísceras ocas. Frequentemente é associada à sensações autonômicas, como náusea, vômito e sudorese. A dor nociceptiva visceral ocorre, por exemplo, em casos de neoplasmas pancreáticos, obstruções intestinais e metástases intraperitoneais.

A dor não nociceptiva é dividida em neuropática e psicogênica. Por definição, dor neuropática é a dor iniciada por lesão ou disfunção do sistema nervoso, resultado da ativação anormal da via nociceptiva (fibras de pequeno calibre e trato espinotalâmico) (MERSKEY; BOGDUK, 1994). Pode ter origem periférica ou central (THURMON; TRANQUILLI.; BENSON, 1999), e qualquer situação que lese ou irrite o tecido neural ou danifique sua função pode ser fonte de dor neuropática (TENG, 2003). Essa dor persiste por longo tempo após o evento precipitante e pode ser ocasionada por sensibilização central. Em pessoas, a dor neuropática é descrita como ardente e penetrante, e os pacientes queixam-se de sensações paroxísticas elétricas (dores lancinantes ou fulgurantes), sendo que estímulos usualmente inócuos podem ser anormalmente percebidos como dolorosos (alodinia) (DUARTE, 1998). Já a dor psicogênica ocorre quando nenhum mecanismo nociceptivo ou neuropático pode ser identificado, e há sinais psicológicos suficientes para estabelecer critérios de distúrbios dolorosos (DUARTE, 1998).

Quanto ao período de duração, classifica-se a dor como aguda ou crônica. A dor aguda tipicamente surge de trauma ou inflamação de tecidos moles, e está relacionada a um processo biológico adaptativo que visa facilitar reparo tecidual e cicatricização. A hipersensibilidade, tanto na área da injúria (hiperalgesia primária) quanto nos tecidos adjacentes (hiperalgesia secundária),contribui para que o processo cicatricial ocorra sem interferências (LAMONT;TRANQUILLI, 2000; JI; WOOLF, 2001; MUIR III; GADAWSKI, 2002)A dor aguda tem início abrupto, conduzida por fibras delta, é bem localizada e termina rapidamente quando o estímulo doloroso é removido. A (THURMON; TRANQUILLI.; BENSON, 1999; WOOLF, 2004).

Já a dor crônica é definida como uma dor que persiste por mais de seis meses (CHONG et al., 2001) e geralmente está vinculada à aspectos emocionais e sofrimento físico, sendo mais difícil de tratar que a dor aguda (KONRAD et al., 2001). É uma dor mal localizada e continua após a remoção do estímulo, sendo conduzida por fibras C (THURMON; TRANQUILLI; BENSON, 1999). Além de simplesmente manifestar-se por longo período de tempo, a dor crônica implica numa síndrome debilitante que possui significativo impacto sobre a qualidade de vida do paciente, caracterizando-se por resposta insuficiente às terapias analgésicas convencionais (KLAUMANN; WOUK; SILLAS, 2008).

\section{Consequências da dor}

Quando a dor assume natureza patológica, suas consequências incluem aumento do risco de infecção, atraso na cicatrização de ferimentos, redução do consumo de água e alimentos, imobilidade e alterações comportamentais e no padrão de sono (TRANQUILLI et al., 2005). A dor não é apenas um incômodo, mas envolve complexas reações fisiológicas, com manifestações autonômicas e psicológicas que levam a imunossupressão, diminuição da perfusão tecidual, espasmo muscular, aumento do trabalho cardíaco e do consumo de oxigênio, alteração da dinâmica respiratória e liberação dos hormônios do estresse, com aumento dos níveis de aldosterona, cortisol e catecolaminas. O processo culmina em elevação do catabolismo e redução do aporte hídrico e alimentar, levando a perda de peso e alteração do equilíbrio nitrogenado. Tais eventos são diretamente relacionados ao aumento de morbidade e mortalidade (BASSANEZI; OLIVEIRA FILHO, 2006).

A dor tratada incorretamente pode produzir efeitos negativos nos sistemas cardiovascular, respiratório, gastrintestinal, neuroendócrino e imune. A elevação secundária nos níveis de cortisol pode prejudicar a cicatrização de feridas e diminuir a eficiência do sistema imune (BIEBUYCK, 1990). A dor ativa o sistema neuroendócrino e causa a liberação de catecolaminas, glucagon, insulina e somatostatina, contribuindo para o aparecimento da caquexia. O aumento na retenção de líquidos leva à ativação do sistema renina-angiotensina, que eleva a pressão arterial e diminui a perfusão renal, e o aumento no consumo de oxigênio pelo miocárdio pode ser deletério para pacientes cardiopatas (LESTER; GAYNOR, 2000).

\section{Etiologia e fisiopatologia da dor neuropática}

Exemplos clássicos de dor neuropática em pessoas incluem neuralgia do trigêmeo, neuralgia pós-herpética e neuropatia periférica (DUARTE, 1998). Cães e gatos podem adquirir dor neuropática em função de condições como trauma acidental e cirúrgico, hérnia inguinal, fraturas pélvicas, amputações ("dor fantasma"), cistite intersticial felina, lesões na medula espinhal, hérnia de disco intervertebral, neuropatias diabéticas e tumores no sistema nervoso central, entre outras. Em procedimentos cirúrgicos, tecido nervoso pode ser inadvertidamente incorporado às suturas, ocasionando dor neuropática de difícil diagnóstico e tratamento. Como prevenção, se houver necessidade de manipulação intensa, tração ou transecção de nervos, indica-se o uso local da associação de lidocaína e bupivacaína, ao menos cinco minutos antes de tais procedimentos (MATHEWS, 2008).

Existem muitas teorias para tentar explicar os mecanismos responsáveis pelo desenvolvimento da dor neuropática, na maioria fundamentadas em modelos neuroquímicos excessivamente teóricos e complexos, de pouco entendimento entre os próprios neurologistas (WOOLF; MANNION, 1999; WOOLF; SALTER, 2000; DEVOR, 2000). Atualmente, o mecanismo mais plausível e cientificamente aceito para explicar a dor neuropática é a geração ectópica de impulsos nervosos às fibras de pequeno calibre do tipo $\mathrm{C}$ e $\mathrm{A} \delta$ (BOSTOCK et al., 2005). Após a lesão nervosa, alguns pacientes desenvolvem alteração na distribuição e conformação de canais iônicos (especialmente canais de sódio) que promovem aumento da excitabilidade dos axonal das fibras finas nociceptivas. Tal excitabilidade muitas vezes é gerada longe do foco da lesão inicial, por descargas ectópicas, mas é capaz de acarretar o surgimento de sinais de características neuropáticas. Não é por acaso que um dos tratamentos mais eficazes para a dor neuropática é o uso dos anticonvulsivantes que agem sobre os canais iônicos, tais como carbamazepina e gabapentina (SCHESTATSKY, 2008). 
Sinais clínicos e diagnóstico da dor neuropática em medicina veterinária

Cães com dor frequentemente alteram seu comportamento interativo, podendo se tornar mais agressivos e resistir à manipulação ou, pelo contrário, se tornando mais tímidos e procurando maior contato com os que o cercam. Ainda que os cães não tenham o mesmo controle motor dos primatas sobre a musculatura facial, certas mudanças em sua expressão facial podem ser indicativas de dor, como alterações no posicionamento das orelhas. Também pode ocorrer alteração na postura corporal, sendo que muitos relutam em deitar-se para proteger a área dolorida, e cães com dor abdominal podem arquear o corpo e apresentar rigidez dorsal (HELLYER; WAGNER, 1998). Os gatos podem perder o hábito de lambedura higiênica, e animais com dor torácica têm frequência respiratória aumentada e inspirações curtas. Outro sinal de dor é a permanência dos olhos abertos com pupilas dilatadas (FANTONI; MASTROCINQUE, 2002). Lambedura ou mordiscamento excessivos ou viciosos, automutilação e presença de alodinia e hiperalgesia no local de uma lesão são considerados as manifestações clínicas mais sugestivas de dor neuropática em animais (YAZBEK, 2008).

A dor é uma experiência individual, e o quanto dessa experiência se traduz em comportamento observável e mensurável no momento da observação clínica depende de variáveis que incluem espécie, linhagem genética dentro da espécie, sexo, massa corporal, condicionamento prévio, características sociais e hierárquicas, saúde sistêmica e condições ambientais (HARDIE, 2002).

Diagnosticar a dor em pacientes incapazes de relatar experiências não é tarefa simples, mas acredita-se que o mecanismo de dor no sistema nervoso dos cães e gatos seja similar ao humano. Assim, se pessoas relatam dor em condições a que os animais também são expostos (condições inflamatórias cirúrgicas e traumáticas, por exemplo), pressupõe-se que sintam o mesmo. Na dor neuropática, o problema diagnóstico é ainda maior, pela dificuldade em identificar quando os animais têm sensações como formigamento, queimação e agulhamento, manifestações comuns da dor neuropática em pessoas. A boa anamnese fornece informações importantes para suspeitar que um paciente esteja apresentando ou não dor neuropática, e é fundamental que o exame físico do paciente deixe de ser apenas uma avaliação de marchas e reflexos, mas inclua a avaliação da nocicepção. Para que o tratamento seja eficaz, é preciso entender os mecanismos que desencadeiam a dor e em que etapa desse processo os fármacos do arsenal terapêutico podem atuar de modo a diminuir a dor neuropática (ORLANDO, 2011).

\section{Tratamento farmacológico da dor neuropática}

Fármacos, técnicas ou métodos usados para prevenir e controlar a dor devem ser adaptados individualmente para cada animal, com base no estado geral de saúde do animal, em suas características comportamentais individuais e nos graus de trauma tecidual e de dor (AVMA, 2001). O interesse pelos mecanismos e tratamento da dor neuropática crônica é crescente, e isso deve resultar em avanços terapêuticos significativos no futuro. Assim, será possível ir além da determinação quanto à eficácia de um determinado tratamen- to, chegando-se à identificação de tratamentos mais efetivos para cada paciente (DWORKIN et al., 2003). Diversos grupos de fármacos vêm sendo empregados no tratamento de dor neuropática em pessoas e animais, com predominância de alguns opioides, antidepressivos e anticonvulsivantes.

Desde tempos imemoriais, o ópio e os seus derivados têm sido empregados como sedativos e analgésicos (DUARTE, 2005), e atualmente são considerados os analgésicos mais potentes, sendo amplamente utilizados em cuidados paliativos, dor neuropática, crônica e oncológica (TEIXEIRA et al., 1999). Chamados inicialmente de narcóticos, conceito e nomenclatura dos opioides têm evoluído (GOZZANI, 1994), e atualmente o grupo inclui todas as substancias naturais, semissintéticas ou sintéticas que reagem com os receptores opioides, seja como agonistas ou antagonistas (DUARTE, 2005). Os analgésicos opioides afetam o processamento e a transmissão dos impulsos em níveis múltiplos do encéfalo e da medula espinhal, interagindo com receptores específicos para inibir a transmissão de sinais dolorosos, da zona medular dorsal para os centros superiores, sendo que tálamo e o córtex cerebral têm alta densidade de receptores opioides. Além dos efeitos moduladores ao nível da medula espinhal, a ativação de receptores centrais inibe a transmissão de sinais dolorosos para centros superiores e, consequentemente, bloqueia a percepção de estímulos dolorosos (BISTNER et al., 2002). Os opióides produzem analgesia quando se ligam aos receptores $\mathrm{Mu}(\mu, \mathrm{OP} 1)$, Kappa $(\kappa, \mathrm{OP} 2)$ e Delta $(\delta, \mathrm{OP} 2)$ distribuídos pelo cérebro, corno dorsal da medula espinhal e nervos sensoriais periféricos e autônomos (NOLAN, 2000; LAMONT, 2008; WAGNER, 2009; VALLEJO et al., 2011). A eficácia analgésica dos opioides pode variar com a espécie e a dosagem empregada, e com tipo, duração e intensidade do estímulo doloroso. Os opioides bloqueiam a transmissão periférica e central da via nociceptiva aferente, e por isso são bastante eficientes no tratamento da dor inflamatória aguda. No entanto, não são igualmente eficazes para todos os tipos de dor, sendo que a resposta da dor neuropática a essa classe de fármacos costuma ser insuficiente ou pouco duradoura (RIBEIRO et al., 2002; BASSANEZI; OLIVEIRA FILHO, 2006).

O tramadol produz analgesia em estados de dor aguda e crônica pela ação sinérgica entre mecanismos opióides e não opióides (SANTOS et al., 2007), por meio da inibição da recaptação neuronal de noradrenalina e serotonina (ALEIXO; TUDURY, 2005). O fármaco apresenta vantagens sobre os opioides tradicionais em função da menor incidência de efeitos adversos (SHIPTON, 2000), possibilidade de administração oral (SHIPTON, 2000; LAMONT; MATHEWS, 2007) e efeito prolongado em comparação à morfina (MARTINS et al., 2010; VETTORATO et al., 2010). É utilizado no manejo de dor moderada à grave, sendo indicado no manejo da dor peri-operatória e da dor crônica em cães, e útil no tratamento de estados de alodinia (TAYLOR; ROBERTSON, 2004). Segundo Dworkin et al. (2010), também é eficaz em diversas síndromes dolorosas neuropáticas.

Os antidepressivos tricíclicos (TCAs) estão entre os fármacos mais eficazes no controle da dor crônica, incluindo a neuropática. Exercem ação sedativa, ansiolítica, miorrelaxante e anti-inflamatória, além de regularizar o sono e melhorar o apetite (MOREIRA, 2005). Apesar de originalmente indicados para tratamento da depressão, os TCAs têm espectro 
de ação relacionado com atividades biológicas que incluem a indução de analgesia em certos estados clínicos e sua eficácia no tratamento da dor neuropática é bem documentada em diversos estudos clínicos (GUIDO, 1991; CAYLEY, 2006). Esse mecanismo de ação não está completamente elucidado, sendo sugerido que o efeito analgésico dos TCAs é mediado pelo bloqueio da noradrenalina e recaptação da serotonina, aumentando os níveis desse neurotransmissor e aumentando a ativação dos neurônios inibitórios descendentes (CLARK, 2000; FIELD; MARTIN, 2008). Esse efeito analgésico ocorre mais rapidamente e em doses mais baixas, em comparação ao tratamento da depressão (FIELD; MARTIN, 2008). Particularmente, a amitriptilina é eficaz para neuropatia diabética e neuralgia pós-herpética em pessoas, e pode ser benéfica para outras síndromes dolorosas neuropáticas (FROMMER et al., 1987). Entretanto, deve ser usada com cautela em função do risco de efeitos adversos (FROMMER et al., 1987), e Hardie (2004) lista sedação, irritação gastrintestinal, psicose e depressão da medula óssea como efeitos colaterais dos TCAs.

Os anticonvulsivantes têm indicação clássica em casos de epilepsia e outros quadros convulsivos. Em cães, são utilizados tanto isoladamente quanto em associação a outros medicamentos, quando a origem das convulsões se deve à alterações metabólicas ou orgânicas (MANNO, 2003; PELLEGRINO, 2003). Os fármacos anticonvulsivantes são classificados como de primeira (carbamazepina e fenitoína) e segunda geração (gabapentina e pregabalina), ambas eficazes no tratamento da dor neuropática. Os agentes de segunda geração são bem tolerados, causam menor sedação e têm menores efeitos secundários (MAIZELS; MCCARBERG, 2005). Anticonvulsivantes apresentam comprovada eficácia no controle da dor de origem neuropática, atuando sobre neurotransmissores e canais iônicos, suprimindo circuitos hiper-reativos da medula e córtex espinhal, estabilizando descargas das vias aferentes primárias. Os anticonvulsivantes exercem seus efeitos analgésicos em canais que estão envolvidos tanto no desenvolvimento da epilepsia como da dor neuropática (TREMONT-LUKATS; MEGEF; BACKONJA, 2000; COSTA, 2009; MARTINS; SOUTO, 2011). Muitas vezes, é necessário usá-los em conjunto com outros "co-analgésicos", como opioides e/ou ATCs (DIAMOND; CONIAM, 1999).

A gabapentina foi sintetizada por meio da inserção de um anel ciclohexano na molécula do ácido gama-aminobutírico (GABA) para facilitar sua entrada no sistema nervoso central, atravessando a barreira hematoencefálica. Inicialmente não tinha uso terapêutico para epilepsia e sim contra espasticidade, mas subsequentemente suas propriedades antiepilépticas ficaram evidentes (GUERREIRO, 2001; LAMONT, 2008). Atualmente, entretanto, sua principal indicação terapêutica é o tratamento de dor (GUERREIRO, 2001), com mecanismo ainda não totalmente esclarecido, supondo-se que esse efeito analgésico seja devido à sua ligação às subunidades pré-sinápticas $\alpha 2-\delta 1$ dos canais de cálcio, que são regulados no gânglio dorsal e na medula espinhal após o estímulo nóxico (LAMONT, 2008). A gabapentina tem sido empregada como adjuvante no controle da dor crônica e neuropática e é altamente biodisponível em cães, sendo metabolizada no fígado e excretada quase exclusivamente pelos rins, com meia-vida de cerca de três a quatro horas e farmacocinética não alterada pelo uso de doses múltiplas (GAYNOR,

\section{9).}

Outros fármacos que podem ser eficazes no alívio da dor neuropática são os bloqueadores de receptores NMDA (cetamina), os agonistas adrenérgicos- $\alpha 2$ (clonidina, dexmedetomidina), os anticolinérgicos (prostigmina), os canabinoides e os inibidores das encefalinases (LOGRASSO; McKELVY, 2003). Nenhum desses fármacos, contudo, é recomendado isoladamente, e além do potencial de efeitos adversos, não são validados por ensaios clínicos bem controlados (FINNERUP et al., 2007).

\section{Relato de Caso}

Relata-se o uso de gabapentina para tratamento e manejo clínico de um caso dor neuropática em uma cadela da raça Schnauzer Miniatura com idade de nove anos e massa corporal de $8,0 \mathrm{~kg}$.

No dia 12 de Fevereiro de 2016 o animal passou por uma consulta de rotina. Na oportunidade também foi feito corte das unhas, seguido do uso tópico de percloreto de ferro, para controle das pequenas hemorragias decorrentes do procedimento. No membro torácico direito (MTD) havia uma unha fraturada longitudinalmente, em avançado processo inflamatório, que foi cortada e higienizada, passando pela mesma cauterização. Em função dessa inflamação, a paciente foi medicada com dose única de $0,2 \mathrm{mg} / \mathrm{kg}$ de meloxicam ${ }^{1}$, administrado por via subcutânea (SC).

No dia 29 de Fevereiro de 2016 a responsável relatou que o animal estava claudicando, e apresentando muita dor nos membros pélvicos (MPs). Ao exame físico apresentava dor articular apenas nos dígitos de ambos os MPs, especialmente o membro pélvico direito (MPD). As articulações falangeanas não estavam edemaciadas, e coluna vertebral e a pelve não apresentavam problemas à palpação, de forma que a suspeita clínica encaminhou-se para "artrite", sem se poder precisar se a alteração era aguda ou crônica. A responsável foi instruída sobre a eventual necessidade de realizar um perfil radiográfico completo, que recusou naquele momento. Assim, foi prescrito o uso oral de meloxicam ${ }^{2}$, na dose de 0,5 mg (1 comprimido) no mesmo dia 29 , seguida de $0,375 \mathrm{mg}$ ( $3 / 4$ comprimido) a cada 24 horas, durante mais seis dias.

No dia 07 de Março de 2016 foi realizado retorno, no qual o animal mantinha o mesmo quadro clínico da semana anterior, e tudo sugeria que houvesse artrite-artrose nos MPs, especialmente MPD. Indicou-se o início do emprego de colágeno bioativo ${ }^{3}$ do tipo 2 preparado em farmácia de manipulação veterinária especializada, por via oral, na dose de uma cápsula de $20 \mathrm{mg}$ ao dia, durante no mínimo 90 dias, e finalmente foi aceita a indicação de realização de radiografias diagnósticas. Adicionalmente, prolongou-se a prescrição de meloxicam oral, até que as imagens radiográficas estivessem disponíveis.

As radiografias pormenorizadas de todo o esqueleto apendicular e axial, tomadas em no mínimo duas exposições, incluindo crânio, pelve e cauda, foram disponibilizadas no dia 09 de Março de 2016. A avaliação dessas imagens não mostrou evidência de comprometimento ósseo ou articular de qualquer tipo, apesar da paciente continuar a demonstrar

\footnotetext{
${ }^{1}$ Melocox $^{\circledR}$, Lab. Eurofarma, São Paulo - SP.

${ }^{2}$ Maxicam ${ }^{\circledR}$ 0,5mg, Lab. Ouro Fino, Cravinhos - SP.

${ }^{3} \mathrm{UC}-\mathrm{II}^{\circledR}$, Farmácia Drogavet, Maringá - PR.
} 
sensibilidade intensa, especialmente à palpação das extremidades traseiras. A partir daí a suspeita principal passou a ser dor neuropática e a responsável foi informada sobre a possibilidade de tratar de forma tentativa com gabapentina.

Além dos dados supracitados, as imagens indicavam também certo índice de retenção fecal, presença de diversos pequenos cálculos na vesícula urinária, e dilatação do lado direito do coração, situação que se coadunava com um sopro mitral identificado por ausculta cardíaca ao exame físico e possibilitou um diagnóstico de insuficiência cardíaca de grau II.

Com base em todos os elementos diagnósticos disponíveis, instituiu-se um protocolo de tratamento que incluiu mudança da alimentação e uso de medicamentos. Quanto à dieta, foi indicado alimento industrializado especial para distúrbios urinários ${ }^{4}$ e uso associado de farelo de aveia constantemente na dieta. Em termos de medicação, prescreveu-se o uso oral de gabapentina ${ }^{5}$ preparada em farmácia de manipulação veterinária especializada (Drogavet $\AA$ ), na dose de $3,75 \mathrm{mg} / \mathrm{kg}$ sendo uma cápsula de $30 \mathrm{mg}$ a cada 12 horas durante no mínimo 90 dias, para tratamento da dor neuropática, e maleato de enalapril ${ }^{6}$ na dose de $0,31 \mathrm{mg} / \mathrm{kg}$ a cada 12 horas, indefinidamente, para manejo da cardiopatia.

Infelizmente, logo após receber a prescrição a responsável teve problemas pessoais que a impediram de solicitar a manipulação da gabapentina, e na madrugada de 16 de Março de 2016 o animal teve um episódio de dor intensa, principalmente nos membros pélvicos, tremendo e vocalizando em desespero. Foi então levada em urgência para ser atendida em um estabelecimento veterinário de plantão, onde não se conseguiu sequer realizar exame físico, sendo somente medicada com cloridrato de tramadol injetável e recebendo uma prescrição do mesmo fármaco por via oral.

No dia 18 de Março de 2016 a paciente passou a receber as cápsulas de gabapentina de acordo com a prescrição, e já no dia seguinte a redução na dor era perceptível para a família. O tratamento persistiu, e no dia 29 de Março de 2016 a recuperação já era classificável como excelente, sem manifestações perceptíveis de dor ou sensibilidade articular em nenhum ponto do corpo, e retorno aos comportamentos e hábitos normais.O tratamento com $30 \mathrm{mg}$ de gabapentina a cada 12 horas foi mantido por mais 20 dias, e a seguir a paciente passou a receber $30 \mathrm{mg}$ a cada 24 horas por mais 20 dias. Depois disso, cessou o uso do medicamento e iniciou-se um período de observação para verificar se havia possibilidade de se considerar a paciente curada.

Em 17 de Maio de 2016, sete dias após a cessação do uso da gabapentina, o animal passou a apresentar novamente sinais de dor articular nos MPs, e passou por nova avaliação clínica. Concluiu-se que seria necessário um tratamento de longo prazo, e retomou-se o uso de $30 \mathrm{mg}$ de gabapentina a cada 12 horas, que persiste desde então, perfazendo seis meses de acompanhamento, período em que o estado geral da paciente foi considerado satisfatório, sem que apresentasse sinais de dor ou desconforto. Definiu-se a manutenção do tratamento por mais seis meses, antes de qualquer redução de doses ou interrupção da administração.

${ }^{4}$ Royal Canin ${ }^{\circledR}$ Urinary, Royal Canin Brasil, Descalvado - SP.

${ }^{5}$ Gabapentina, Farmácia Drogavet, Maringá - PR

${ }^{6}$ Petpril ${ }^{\circledR}$, Agener-União, Embu-Guaçu - SP.

\section{Discussão e Conclusão}

A dor é um fenômeno importante na Medicina Veterinária, pois compromete diretamente a recuperação dos doentes e, em situações extremas, pode até conduzir à morte (CASTRO, 2008).O interesse pelos mecanismos e tratamento da dor neuropática crônica é crescente, e isso deve resultar em avanços terapêuticos significativos no futuro. Assim, será possível ir além da determinação quanto à eficácia de um determinado tratamento, chegando-se à identificação de tratamentos mais efetivos para cada paciente (DWORKIN et al., 2003).A dor neuropática é conceituada como sendo uma "dor por consequência direta de lesão ou doença afetando o sistema somato-sensitivo periférico ou central" (TREED et al., 2008).

Segundo Schestatsky (2008) a dor neuropática costuma responder de insuficiente aos analgésicos comuns, sendo os fármacos antidepressivos tricíclicos e anticonvulsivantes as principais indicações no tratamento desse tipo de dor, tanto de origem central quanto periférica. Os anticonvulsivantes representam importante opção no tratamento da dor neuropática (JENSEN, 2002), e segundo Figueiredo e Flôr (2011) são indicados para alívio da dor neuropática quando existir alodinia e hiperalgesia.

A gabapentina, substância análoga ao GABA com boa penetração na barreira hematoencefálica, foi aprovada nos EUA no final de 1993, para tratamento aditivo de distúrbios convulsivos (MARCOLIM; TATSCH, 2000) e nos últimos anos vem sendo usada de forma corrente no tratamento da dor neuropática (JENSEN, 2002; SCHATZBERG et al., 2009).

Estudos em cobaias comprovam que a gabapentina reduz consideravelmente os sinais da dor neuropática como hiperalgesia e alodinia (MAO; CHEN, 2000). Em ratos, a gabapentina administrada pela via oral ou intratecal inibe a transmissão da dor inflamatória e reduz hiperalgesia causada por injuria nervosa periférica e queimadura (GAYNOR, 2009). Investigações realizadas em pessoas, incluindo estudos duplos-cegos, atestam a eficácia da gabapentina na redução da dor associada à neuralgias como a do trigêmeo, pós-herpética e diabética (SCHATZBERG et al., 2009), sem os efeitos colaterais e o abuso geralmente associado aos opioides (BUCCAFUSCO et al., 2010).

Embora doses para cães e gatos não tenham sido estabelecidas de forma laboratorial e não existam estudos controlados ou baseados em evidências nessas espécies utilizando gabapentina, já se investigou seu uso como antiepilético em cães, com doses variando entre 800 e 1500 mg diárias. As doses iniciais variam de 2,5 a 10,0 mg/kg, por via oral, a cada oito ou 12 horas, podendo chegar a $50 \mathrm{mg} / \mathrm{kg}$. Alguns clínicos utilizaram até mesmo doses maiores e com maior frequência (GAYNOR, 2009). Yazbek e Martins (2011) preconizam a administração da gabapentina na dose de 3-10 mg/ $\mathrm{kg}$ a cada oito horas, e Tranquilli et al. (2005) indicam dose canina de $5-10 \mathrm{mg} / \mathrm{kg}$, em administrações a cada 12 horas. Neste caso foi empregada a dose de $3,75 \mathrm{mg} / \mathrm{kg}$ a cada 12 horas, corroborando as indicações de Gaynor (2009).

Ainda não é possível fazer recomendações científicas específicas de protocolos de tratamento para animais de estimação, doses e indicação, mas com o consentimento dos responsáveis, gabapentina e pregabalina são cada vez mais 
utilizadas por médicos veterinários na tentativa de controlar melhor a dor neuropática crônica, de origem maligna ou não, em animais de companhia (LAMONT; MATHEWS, 2013).

A gabapentina é absorvida pelo trato gastrintestinal por transporte ativo, sendo que 3\% se ligam às proteínas plasmáticas (MACEDO, 2009). É metabolizada rapidamente por meio de uma esterase hepática, com excreção renal (LASCELLES, 2005). Sua absorção oral é bastante eficaz e rápida, atingindo concentração plasmática máxima em media 1,3 horas após a administração (KUKANICH; COHEN, 2011). É um fármaco de ação curta, com meia-vida aproximada de 3,3 horas, e tem baixo potencial para interações farmacológicas, já que não se liga a proteínas e é totalmente excretada pelos rins (PODELL, 2004; GOVENDIR et al., 2005). Até o momento, não foram descritas complicações hematológicas, hepáticas e dermatológicas significantes causadas pela gabapentina, e seus efeitos adversos têm relação direta com a dose, sendo mais comuns sonolência ( $20 \%$ dos pacientes), ataxia (17\%) e nistagmo (15\%) (SEGAL; RORDORF, 1996), relatando-se ainda inapetência, fadiga, ganho de peso, emese e sialorreia (MUÑANA, 2013). Testes clínicos referentes à administração crônica em pessoas informam efeitos colaterais em $25 \%$ dos pacientes, incluindo sonolência, fadiga e ganho de peso (STEFAN; FEUERSTEIN, 2007; GAYNOR, 2009), e todos esses efeitos cessam com a interrupção do uso da gabapentina (YACUBIAN, 2002). Segundo Grubb (2010) o efeito secundário mais comum é a sedação, que pode ser controlada com ajuste posológico.

Fármacos de uso contínuo exigem monitorização clínica constante dos animais tratados, para evitar sobrecarga dos sistemas de metabolização e excreção (MUÑANA, 2013). No caso apresentado houve monitorização laboratorial periódica com avaliação do hemograma e provas de função hepática (alanina aminotransferase e fosfatase alcalina) e renal (ureia e creatinina), não se observando quaisquer alterações significativas.

Finalmente, é importante tecer suposições sobre a causa eventual do processo álgico que acometeu a paciente. É possível que o corte das unhas e/ou o uso de percloreto de ferro para controle das pequenas hemorragias decorrentes do procedimento tenham atuado como agente desencadeador. Entretanto, o corte de unhas em cães é procedimento extremamente frequente em clínicas, petshops e nos próprios domicílios, considerado de alta segurança. Quanto ao percloreto de ferro, é um agente hemostático de uso corriqueiro em diversas situações, e não existem registros de que cause quadros como o aqui discutido.

No caso apresentado, o uso da gabapentina mostrou-se uma opção terapêutica de fácil acesso e utilização, que foi eficaz no manejo clínico da dor neuropática em longo prazo, pois houve a redução dos sinais de dor e melhora na qualidade de vida da paciente, sem que se observassem efeitos adversos.

\section{Referências}

AGS (American Geriatric Society) Panel on Persistent Pain in Older Persons. The Management of Persistent Pain in Older Persons. Journal of the American Geriatric Society (JAGS), v. 50, n. 6, p. 5-24, 2002.

ALEIXO, G. A. S.; TUDURY, E. A. Utilização de opioides na analgesia de cães e gatos. Veterinária Notícias, Uberlândia, v. 11, n. 2, p. 31-42, 2005.

ALMEIDA, T. P. et al. Classificação dos processos dolorosos em medicina veterinária - revisão de literatura. Revista Veterinária em Foco, Canoas, v. 3, n. 2, p. 107-118, 2006.

AVMA (American Veterinary Medical Association) adopts position regarding animal pain. Journal of the American Veterinary Medical Association, v. 218, p. 1694, 2001.

BACKONJA, M. M. Defining neuropathic pain. Anesthesia \& Analgesia, Baltimore, v. 97, p. 785-790, 2003.

BASSANEZI, B. S. B.; OLIVEIRA FILHO, A. G. D. E. Analgesia pós-operatória. Revista do Colégio Brasileiro de Cirurgia, Rio de Janeiro, v. 33 n. 2, p. 116-122, 2006.

BENNET, M. I. et al. Can pain can be more or less neuropathic? Comparison of symptom assessment tools with ratings of certainty by clinicians. Pain, Amsterdam, v. 122, p. 289-294, 2006.

BIEBUYCK, J. F. The metabolic response to stress: an overview and update. Anesthesiology, New York, v. 73, p. 308-327, 1990.

BISTNER, S. I.; FORD, R. B.; RAFFE, M. R. Manual de procedimentos veterinários e tratamento emergencial. 7. ed. São Paulo: Roca, 2002. 11-14 p.

BOSTOCK, H. et al. Temperature- dependent double spikes in C-nociceptors of neuropathic pain patients. Brain. v. 128, p. 2154 2163, 2005 .

BUCCAFUSCO, J. J. et al. Treatments for neuropathic pain differentially affect delayed matching accuracy by macaques: Effects of amitriptyline and gabapentin. Pain, v. 148, n. 3, p. 446$453,2010$.

CAPONERO, R.; VIEIRA, D. E.; TEIXEIRA, M. J. Dor no Doente com Câncer. Prática Hospitalar, 2001.

CASTRO, D. S. Comparação entre o efeito analgésico da morfina e do tramadol epidural em gatos (Felis catus domesticus). 2008. 48 f. Dissertação (Mestrado em Ciências Veterinárias) - Universidade Federal Rural do Rio de Janeiro, Rio de Janeiro, 2008

CAYLEY, W. E. Antidepressants for the treatment of neuropathic pain. American Family Physician (AFP), v. 73, n. 11, p. 19332011, 2006

CHONG, G. S. et al. Chronic Pain and Self - efficacy: The Effects of Age, Sex, and Chronicity. Pain Pratice, Ohio, v. 1, n. 4, p. 338-343, 2001.

CLARK, M. R. Pharmacological treatments for chronic nonmalignat pain. International Review of Psychiatry, v. 12, p. $148-156,2000$

COSTA, C. M. C. Dor neuropática.In: NETO, A. O. et al. Dor: princípios e prática. Porto Alegre: Artmed, 2009. p. 495-509.

DEVOR, M. Neuropathic pain: what do we do with all these theories? Acta Anaesthesioloca Scandinavica, v. 45, p. 11211127,2000

DIAMOND, A. W.; CONIAM, S. W. Controle da dor crônica. Lisboa: Climepsi Editores, 1999.

DUARTE, D. F. Uma breve historia do ópio e dos opioides. Revista Brasileira de Anestesiologia, v. 55, n. 1, p. 135-146, 
2005.

DUARTE, R. A. Classificação da Dor. In: KANNER, R. Segredos em clínica de dor. Porto Alegre: Artmed, 1998. p. 22-24.

DWORKIN, R. H. et al. Advances in Neuropathic Pain: Diagnosis, Mechanisms, and Treatment Recommendations. Archives of Neurology, v. 60, n. 11, p. 1524-1534, 2003

DWORKIN, R. H. et al. Recomendações para o Tratamento Farmacológico da Dor Neuropática: Visão Global e Atualização da Literatura. Mayo Clinic Proceedings, v. 85, p. 3-14, 2010.

FANTONI, D. F.; MASTROCINQUE, S. Fisiopatologia e Controle da Dor. In: FANTONI, D. T.; CORTOPASSI, S. R. G. Anestesiologia em cães e gatos. São Paulo: Roca, 2002. p. 323-336.

FANTONI, D. T.; MASTROCINQUE, S. Analgesia Preventiva, mito ou fato? Clínica Veterinária, n. 49, p. 24-32, 2004.

FANTONI, D. T.; MASTROCINQUE, S. Fisiopatologia e controle da dor aguda In: Anestesia em cães e gatos. 2 . ed. São Paulo: Roca, 2010. p. 521-544.

FIELD, H. L.; MARTIN, J. B. Pain: Pathophysiology and management. In: FAUCI, A. C. et al. Harrison's principles of internal medicine. 17. ed. USA: McGraw Hill, 2008. p. 71-76.

FIGUEIREDO, R. C. C.; FLÔR, P. B. Tratamento do dor crônica. In: FANTONI, D. T. Tratamento da dor na clínica de pequenos animais. São Paulo: Elsevier, 2011. p. 361-382.

FINNERUP, N. B. et al. An evidence-based algorithm for the treatment of neuropathic pain.Medscape General Medicine, v. 9, n. 2 , p. $36,2007$.

FROMMER, D. A. et al. Tricyclic antidepressant overdose. Journal of the American Medical Association (JAMA), v. 257, p. 521-526, 1987

GAYNOR, J. S. Other Drugs Used to Treat Pain. In: GAYNOR, J. S.; MUIR, W. W. Handbook of veterinary pain management. 2. ed. Missouri: Mosby, 2009. p. 260-276.

GOVENDIR, M.; PERKINS, M.; MALIK, R. Improving seizure control in dogs with refractory epilepsy using gabapentin as an adjunctive agent. Australian Veterinary Journal, v. 83, n. 10, p. 602-608, 2005

GOZZANI, J. Opioides e antagonistas. Revista Brasileira de Anestesiologia, v. 44, p. 65-73, 1994.

GRUBB, T. What do we really know about the drugs we use to treat chronic pain? Topics in Companion Animal Medicine, v. 25 , p. $10-19,2010$

GUERREIRO, C. A. M. Tratamento medicamentoso das epilepsias com novas drogas e complicações de epilepsias crônicas. 2001. Dissertação (Livre-Docência) - Faculdade de Ciências Médicas, Universidade Estadual de Campinas, Campinas, 2001.

GUIDO, M.The use of antidepressants in the treatment of chronic pain. Drugs, v. 42, p. 730-748, 1991.

HARDIE, E. M. Dor: controle. In: ETTINGER, S. J.; FELDMAN E. C. Tratado de medicina interna veterinária: doenças do cão e do gato. 5. ed. Rio de Janeiro: Guanabara Koogan, 2004. p. 24-26
HARDIE, E. M. Reconhecimento do Comportamento Doloroso em Animais. In: HELLEBREKERS L. J. Dor em animais. São Paulo: Manole, 2002. p. 49-79.

HELLEBREKERS, L. J. A dor em animais In: animais. São Paulo: Manole, 2002. p. 11-17.

Dor em

HELLYER, P. W.; WAGNER, A. E. Tratamento da Dor nas Emergências. In: WINGFIELD, W. E. Segredos em Medicina Veterinária. Porto Alegre: Artmed, 1998. p. 117-124.

JENSEN, T. S. Anticonvulsants in neuropathic pain: rationale and clínical evidence. European Journal of Pain, v. 6, p. 61-68, 2002.

JI, R. R.; WOOLF, C. J. Neuronal plasticity and signal transduction in nociceptive neurons: implications for the initiation and maintenance of pathological pain. Neurobiology of Disease, Massachusetts, v. 8, n. 1, p. 1-10, 2001.

JOHNSTON, S. A. Dor: identificação In: ETTINGER, S. J.; FELDMAN, E. C. Tratado de medicina interna veterinária: Doenças do cão e do gato. 5. ed. Rio de Janeiro: Guanabara Koogan, 2004. p. 21-23.

KITCHELL, R. Problems in defining pain and peripheral mechanisms of pain. Journal of the American Veterinary Medical Association, v. 191, p. 1195-1199, 1987.

KLAUMANN, P. R.; WOUK, A. F. P. F.; SILLAS, T.

Patofisiologia da dor. Archives of Veterinary Science, Curitiba, v. 13, n. 1, p. 1-12, 2008

KONRAD et al. Dor, Fisiopatologia e Tratamento. In: LIANZA, S. Medicina de Reabilitação. 3. ed. Rio de Janeiro: Guanabara Koogan, 2001. p. 137-150.

KUKANICH, B.; COHEN, R. L. Pharmacokinetics of oral gabapentin in greyhound dogs. Veterinary Journal, v. 187, n. 1, p. 133-135, 2011.

LAMONT, L. A. Multimodal pain management in veterinary medicine: the physiologic basis of pharmacologic therapies. Veterinary Clinics of North America: Small Animal Practice, v. 38 , n. 6 , p. $1173-1186,2008$

LAMONT, L. A.; MATHEWS, K. A. Opioides, anti-inflamatórios não esteroidais e analgésicos adjuvantes. In: TRANQUILLI, W. J.; THURMON, J. C.; GRIMM, K. A. LUMB \& JONES: Anestesiologia e analgesia veterinária. 4. ed. São Paulo: Roca, 2013. p. $270-304$

LAMONT, L. A.; MATHEWS, K. A. Opioids, Nonsteoidal antiinflammatories and analgesic adjuvants. In: TRANQUILLI, W. J. et al. Veterinary Anesthesia and Analgesia, 4.ed. Iowa: Blackwell Publishing, 2007. p. 241-271

LAMONT, L. A.; TRANQUILLI, W. J. Physiology of pain. Veterinary Clinics of North America: Small Animal Practice, Philadelphia: Saunders, v. 30, n. 4, p. 703-728, 2000.

LAMONT, L. A.; TRANQUILLI, W. J.; GRIMM, K. A. Physiology of pain. Veterinary Clinics of North America, v. 30, n. 4, p. 703-728, 2000.

LASCELLES, B. D. X. Manejo da dor crônica em pequenos animais: terapia multimodal. In: OTERO, P. Dor-Avaliação e tratamento de pequenos animais. Buenos Aires: Interbook, 2005. p. $142-155$.

LESTER, P.; GAYNOR, J. S. Management of cancer pain. Veterinary Clinics of North America: Small Animal Practice, 
Colorado, v. 30, n. 4, p. 951-966, 2000.

LOGRASSO, P.; MCKELVY, J. Advances in pain therapeutics. Current Opinion in Chemical Biology, v. 7, p. 452-456, 2003.

LORENZ, M. D.; KORNEGAY, J. N. In: Neurologia veterinária. São Paulo: Manole, 2006. p. 345-353.

MACEDO, D. D. P. Drogas antiepilépticas e psicotrópicas. In: ALVES NETO, O. et al. Dor: princípios e prática. Porto Alegre: Artmed, 2009. p. 1108-1114.

MAIZELS, M.; MCCARBERG, B. Antidepressants and Antiepleptic Drugs for Chronic Non-Cancer Pain. American Family Physician, v. 71, n. 3, p. 483-490, 2005.

MALANGA, G.; PASTER, Z. "Update on managing chronic pain in the elderly". The Journal of Family Practice, v. 56, n. 12, p. 11-6, 2007.

MANNO, E. M. New management strategies in the treatment of status epilepticus. Mayo Clinic Proceedings, Rochester, v. 78, n. 4, p. 508-518, 2003.

MAO, J.; CHEN, L. L.; Gabapentin in pain management. Anesthesia and Analgesia, v. 91, n. 3, p. 680-687, 2000.

MARCOLIN, M. A.; TATSCH, M. F. Gabapentina: farmacologia uso clínico e interações farmacológicas. Revista de Psiquiatria Clínica, v. 27, n. 4, p. 237-243, 2000.

MARTINS, A. T. L.; SOUTO, M. T. Antidepressivos e anticonvulsivantes. In: FANTONI, D. T. Tratamento da dor na clínica de pequenos animais. Rio de Janeiro: Elsevier, 2011. p. 195-202.

MARTINS, T. L. et al. Comparison of the effects os tramadol, codeine, and ketoprofen alone or in combination on postoperative pain and on concentrations of blood glucose, serum cortisol, and serum interleukin- 6 in dogs undergoing maxillectomy or mandibulectomy. American Journal of Veterinary Research, v. 71, n. 9, p. 1019-1026, 2010

MATHEWS, K. A. Dororigem e efeito.In: RABELO, R. C.; CROWE Jr., D. T. Fundamentos de terapia intensiva veterinária em pequenos animais - Condutas no paciente crítico. Rio de Janeiro: L. F. Livros, 2005. p. 519-527.

MATHEWS, K. A. Neuropathic pain in dogs and cats: if only they could tell us if they hurt. The Veterinary Clinics of North America: Small Animal Practice, Philadelphia, v. 38, p. 1365 1414, 2008.

MERSKEY, H.; BOGDUK, N. Classification of chronic pain. International Association for the Study of Pain (IASP) Press, Seattle, 1994.11-12 p.

MOREIRA, J. C. Controle da dor em UTI: identificação da dor através do comportamento. In: RABELO, R. C.; CROWE JUNIOR, D. T. Fundamentos de terapia intensiva veterinária em pequenos animais: Condutas do paciente crítico. Rio de Janeiro: L. F. Livros de Veterinária, 2005. p. 495-506.

MUIR III, W. W.; GADAWSKI, J. E. Cardiovascular effects of a higher dose of romifidine in propofol anesthetized cats. American Journal of Veterinary Research, Colorado, v. 63, n. 12, p. 12411246, 2002.
MUIR III, W. W.; GAYNOR, J. S. Pain Behaviours. In: MUIR III, W. W.; GAYNOR, J. S.Handbook of veterinary pain management. St. Louis: Mosby, 2009. p. 62-77.

MUÑANA, K. R. Management of refractory epilepsy. Topics in Companion Animal Medicine, v. 28, n. 2, p. 67-71, 2013.

NOLAN. Pharmacology of analgesic drugs. In: FLECKNELL, A. P.; WATERMAN, A. E. Pain Management in Animals. London: W.B. Saunders, 2000. p. 21-52.

ORLANDO, C. F. P. Mecanismos da dor neuropática (Revisão de literatura). 2011. Seminário - Programa de Pós-Graduação em Ciência Animal da Escola de Veterinária e Zootecnia da Universidade Federal de Goiás (UFG). Nível: Doutorado. Goiânia, 2011.

PATEL, N. B. Physiology of pain. In: KOPF, A.; PATEL, N. $B$. Guide to pain management in low-resource settings. Amsterdam: Internacional Association for the Study of Pain, 2010. p. $13-18$.

PELLEGRINO, F. Epilepsia e síndromesepilépticas. In: PELLEGRINO, F. C.; SURANITI, A.; GARIBALDI, L. Síndromes neurológicas em cães e gatos. São Paulo: Interbook, 2003. p. 192-215.

PISERA, D. Fisiologia da dor. In: OTERO, P. E. Dor: Avaliação e tratamento em pequenos animais. São Paulo: Interbook, 2005. p. $30-74$.

PODELL, M. S. In: PLATT, S. R.; OLBY, N. J. BSAVA manual of canine and feline neurology. 6. ed. Dorset: British Small Animal Veterinary Association, 2004. p. 97-112.

RIBEIRO, S.; SCHMIDT, A. P.; SCHMIDT, S. R. G. O uso de opioides no tratamento da dor crônica não oncológica: o papel da metadona. Revista Brasileira de Anestesiologia, v. 52, n. 5, p. 644-651, 2002

SANTOS, A. C. D. et al. Estudo dos efeitos analgésicos e das concentrações plasmáticas do tramadol em cadelas submetidas aovario-salpingo-histerectomia. Study of analgesic effects and plasmatic concentrations of tramadol in female dogs submitted to ovaryhisterectomy. Acta Scientiae Veterinariae, v. 35, p. 339341, 2007.

SCHAIBLE, H. G. Pathophysiology of pain. Der Orthopäde. v. 36, n. 1, p. 8-16, 2006.

SCHATZBERG, A. F.; COLE, J. O.; DEBATTISTA, C. Estabilizadores de humor. In: SCHATZBERG, A. F.; COLE, J. O.; DEBATTISTA, C. Manual de psicofarmacologia clínica. 6. ed. Porto Alegre: Artmed, 2009. cap. 5.

SCHESTATSKY, P. Definição, diagnóstico e tratamento da dor neuropática. Revista do Hospital de Clínicas de Porto Alegre, v. 28 , n. 3, p. $177-187,2008$

SEGAL, A. Z.; RORDORF, G. Gabapentin as a novel treatment for postherpetic neuralgia. Neurology, v. 46, p. 1175-1176, 1996.

SHIPTON, E. A. Tramadol - present and future. Anaesthesia and Intensive Care, v. 28, p. 363-374, 2000.

SILVA, C. R. A. Métodos de aferição de pressão arterial em cães anestesiados com propofol e mantidos por halotano. 2010. 
45f. Dissertação (Mestrado em Ciência Animal) - Universidade Federal do Piauí, Piauí, 2010.

SOUSA, F. A. E. F. Dor: o quinto sinal vital. Revista LatinoAmericana de Enfermagem, v. 10, n. 3, p. 446-447, 2002.

SOUZA, H. J. M. et al. Gatos: analgesia pós-operatória. Nosso Clínico, n. 22, p. 8-12, 2001.

STEFAN, H.; FEUERSTEIN, T. J. Novel anticonvulsivant drugs. Pharmacology \& Terapeutics, v. 113, p. 165-183, 2007.

STOELTING, R. K. Pharmacology \& physiology in anesthesic practice, 3.ed. Philadelphia: Lippincott-Raven, 1999. p. 814.

TAYLOR, P. M.; ROBERTSON, S. A. Pain management in cats - Past, present and future. Part 1 . The cat is unique. Journal of Feline Medicine and Surgery, v. 6, p. 313-320, 2004.

TEIXEIRA, M. et al. Tratamento Multidisciplinar do doente com dor. In: CARVALHO, M. Dor: um estudo multidisciplinar. São Paulo: Summus, 1999.

TENG, J. Neuropathic Pain: Mechanisms and Treatment Options. Pain Practice, Ohio-EUA, v. 3, p. 8-21, 2003.

THURMON, J. C.; TRANQUILLI, W. J.; BENSON, J. G. Essentials of Small Animal. Anesthesia \& Analgesia. BaltimoreUSA: Lippincoott Williams \& Wilkins, 1999. 28-60 p.

TRANQUILLI, et al. Técnicas analgésicas. In: Tratamento da dor na clínica de pequenos animais. São Paulo: roca, 2005. p. 323-345.

TRANQUILLI, W. J. Fisiologia da Dor Crônica. In: GREENE, S. A. Segredos em anestesia veterinária e manejo da dor. Porto Alegre: Artmed, 2004. p. 421-423.

TREED, R. D. et al. Neuropathic pain: Redefinition and a grading system for clinical and research purposes. Neurology, v. 70, p. 1630-1635, 2008.

TREMONT-LUKATS, I. W.; MEGEFF, C.; BACKONJA, M. M. Anticonvulsants for neuropathic pain syndromes: mechanisms of action and place in therapy. Drugs, v. 60, n. 5, p. 1029-1052, 2000.

VALLEJO, R.; BARKIN, R. L.; WANG, V. C. Pharmacology of opioids in the treatment of chronic pain syndromes. Pain Physician, v. 14, n. 4, p. 343-360, 2011.

VETTORATO, E. et al. Pharmacokinetics and efficacy of intravenous and extradural tramadol in dogs. Veterinary Journal, v. 183 , n. 3 , p. $305-310,2010$

WAGNER, A. Opioids. In: MUIR III, W. W.; GAYNOR, J. S. Handbook of veterinary pain management. St. Louis: Mosby, 2009. p. 163-182.

WOOLF, C. J. Pain: moving from symptom control toward mechanism-specific pharmacologic management. Annals of Internal Medicine, v. 140, n. 6, p. 441-451, 2004.

WOOLF, C. J.; MANNION, R. J. Neuropathic pain, aetiology, symptoms, mechanisms and management. Lancet, v. 353, n. 9168, p. 1959-1964, 1999.
WOOLF, C. J.; SALTER, M. W. Neuronal plasticity: increasing the gain in pain. Science, v. 288, p. 1765-1768, 2000.

YACUBIAN, E. M. T. Tratamento da epilepsia na infância. Jornal de Pediatria, v. 78, n. 1, p. 19-17, 2002.

YAZBEK, K. V. B. Avaliação da dor e da qualidade de vida em cães com câncer. Revista Dor. São Paulo-SP, v. 9, n. 3, p. 12971304, 2008.

YAZBEK, K. V. B.; MARTINS, T. L. Tratamento da dor oncológica. In: FANTONI, D. T. F. Tratamento da Dor na Clínica de Pequenos animais. São Paulo: Elsevier, 2011. p. $383-$ 395.

Recebido em: 29.10.2017 Aceito em: 27.03.2018 